\title{
Pulmonary arterial hypertension and the Enigma code of smouldering inflammation
}

\author{
Jose Gomez-Arroyo ${ }^{1}$, Antonio Abbate ${ }^{2}$ and Norbert F. Voelkel ${ }^{3}$ \\ Affiliations: ${ }^{1}$ Dept of Internal Medicine, University of Cincinnati, Cincinnati, OH, USA. ${ }^{2}$ Pauley Heart Center, \\ Virginia Commonwealth University, Richmond, VA, USA. ${ }^{3}$ School of Pharmacy, Virginia Commonwealth
} University, Richmond, VA, USA.

Correspondence: Norbert F. Voelkel, School of Pharmacy, Virginia Commonwealth University, 1220 E Broad St, MMRB, 6th floor, Richmond, VA 23298, USA. E-mail: nfvoelkeldgmail.com

@ERSpublications

Anti-interleukin-1 treatment for pulmonary arterial hypertension: are we there yet? http://ow.ly/8irv300EXoo

More than any other cytokine family, the [interleukin]-1 family is closely linked to innate inflammatory and immune responses.

Dinarello [1]

"What causes fever?" was the question that prompted the work that led to the discovery of the "leukocyte pyrogen", eventually named interleukin (IL)-1, in the late 1970s [1]. However, despite the rapid understanding of innate and adaptive immune responses and inflammation during the 1980s, it was not until 1995 that HumberT et al. [2] reported that both IL-1 and IL-6 levels were increased in the plasma of patients with idiopathic pulmonary arterial hypertension (PAH), thus revealing an inflammatory component in the pathogenesis of severe PAH. Ever since the first report by HuMBERT et al. [2], the role of inflammation as a cause or consequence of PAH has remained controversial [3] and this ongoing debate has perhaps prevented the pragmatism to design clinical trials to test novel anti-inflammatory therapies in patients with PAH.

In the current issue of the European Respiratory Journal, PARPALEIX et al. [4] provide one more mechanistic piece of evidence to solve the enigma of aseptic inflammation in PAH and offer another rationale to justify the clinical evaluation of IL-1-based treatment strategies for PAH. The authors demonstrated that the levels of the IL-1 receptor 1 (IL1R1) protein and its adaptor protein MyD88, required for downstream signalling, are increased in the lung tissue samples from patients with idiopathic PAH. They also showed that in mice with chronic hypoxia-induced $\mathrm{PAH}$ and in rats with monocrotaline-induced $\mathrm{PAH}$, treatment with anakinra, a recombinant IL1R1 antagonist, decreased pulmonary vascular muscularisation, right ventricular systolic pressure and right ventricular hypertrophy. The study by PARPALEIX et al. [4] confirmed earlier studies that had demonstrated the increased expression of IL- $1 \alpha$ and IL-1 $\beta$ protein levels in monocrotaline-induced pulmonary hypertensive rat lungs and that chronic treatment with anakinra prevented the development of monocrotaline-induced PAH [5]. To further assess causality, PARPALEIX et al. [4] challenged both IL1R1 and MyD88 knock-out mice with chronic hypoxia, and showed that genetic ablation of the IL-1 $\beta$ canonical signalling machinery ameliorated the pulmonary vascular response to hypoxia. In vitro, they showed how pulmonary vascular smooth muscle cells treated with anakinra had reduced IL-1 $\beta$-induced cell proliferation, NF- $\kappa$ B activation (a major inflammatory regulator) and IL-6 expression (an IL-1 $\beta$-inducible cytokine linked to increased mortality in PAH [6, 7]). Moreover, supernatants from M1-polarised macrophages resulted in pulmonary vascular smooth muscle cell proliferation, partly via IL1R1/MyD88 signalling. However, some questions remain unanswered. For example, 1) what is the mechanism whereby

Received: May 182016 | Accepted: May 262016

Conflict of interest: Disclosures can be found alongside this article at erj.ersjournals.com

Copyright @ERS 2016 
IL-1 $\beta$ induces vascular smooth muscle cell growth? 2) Are there additional MyD88-dependent cytokines involved in the development of hypoxic pulmonary hypertension? 3) How much of the effect of anakinra resulted from blocking IL-1 $\alpha$ ?

The work of PARPALEIX et al. [4], along with the publication by Tian et al. [8], suggests that macrophages may play a significant role in the still enigmatic signalling cascades of inflammatory reactions involved in the pathogenesis of PAH. Whereas the work by TIAN et al. [8] discovered a novel action for macrophage-released leukotriene B4 as an inducer of endothelial cell apoptosis, the work by PARPALEIX et al. [4] suggests that macrophages may also contribute to pulmonary vascular inflammation and proliferation, at least partly via IL-1/IL1R1 signalling. Thus, it appears that both the "leukocyte pyrogen" IL-1 and the "leukocyte-triene" eicosanoid LTB4 are elements of a code that we need to decipher in order to understand the multicellular interactions between macrophages, endothelial cells and other immune cells, which culminate in pulmonary vascular disorders.

While the focus of research in the setting of PAH has been on IL-1 $\beta$, little is known about the role of IL- $1 \alpha$; however, it should not be overlooked because "all autoimmune diseases have a significant inflammatory component that is due to production of both of IL-1 $\beta$ as well as IL-1 $\alpha$ from myeloid cells, particularly macrophages) [...] B cells produce IL-1 $\beta$ and there is IL-1 $\alpha$ from T cells" [1]. IL-1 $\alpha$ is an "alarmin" [9], a DNA-damage sensor [10] and, most importantly, it is highly abundant in endothelial cells [11]. It has been postulated that apoptosis of pulmonary microvascular endothelial cells may be the critically important initiating event in PAH pathogenesis [12] and it is now known that endothelial apoptotic bodies contain IL- $1 \alpha$ [11] that, upon release, could contribute to vascular remodelling. Now that both IL-1 $\beta$ [4] and IL- $1 \alpha$ [5] have been found to be elevated in the lung tissue from pulmonary hypertensive humans and rodents, we should attempt to understand the potential nonredundant roles of these two cytokines in lung vascular diseases. For instance, in murine models of inflammatory bowel disease, IL- $1 \alpha$ mediates inflammation whilst IL-1 $\beta$ mediates healing $[13,14]$; a similar phenomenon could occur in the lung circulation. Nevertheless, IL- $1 \alpha$ induces IL- $1 \beta$, and both IL- $1 \alpha$ and IL- $1 \beta$ share a single type I signalling receptor, making it difficult to dissect the role of the individual cytokines in vivo [14].

Increasing evidence has suggested that high levels of IL-1 $\beta$ predict a worse outcome in patients with severe $\mathrm{PAH}[5,6,15]$. However, despite its prognostic value, no prospective study has explored whether treatment with PAH-specific pharmacotherapies lowers IL-1 $\beta$ or whether lowering cytokine levels can affect survival. Anakinra has been proven to be effective in treating multiple autoinflammatory as well as autoimmune conditions [1]. Among clinical trials, there are some examples of small cohort studies in patients with acute myocardial infarction [16] and acute decompensated or chronic heart failure [17, 18] that have begun exploring the treatment effects of anakinra in cardiovascular diseases, and another large controlled clinical trial is presently underway to probe the inflammatory underpinnings of atherosclerotic diseases $[19,20]$. Perhaps these studies could provide a blue print for the design of IL-1-targeting PAH trials. We believe that pre-clinical PAH trials, like the current study by PARPALEIX et al. [4], should no longer be seen only as academic, l'art pour l'art exercises. Instead, they should lower the threshold for the design and conduct of clinical pilot trials. To us, it appears the time is right for the start of anti-IL PAH trials. The safety profile of anakinra has been assessed and found to be acceptable [21], and thus the risk/ benefit balance may be tilted towards potential benefits.

\section{Acknowledgement}

The authors wish to thank Charles A. Dinarello (University of Colorado, Denver, CO, USA) for his critical reading of this manuscript.

\section{References}

1 Dinarello CA. An expanding role for interleukin-1 blockade from gout to cancer. Mol Med 2014; 20: Suppl. 1, S43-S58.

2 Humbert M, Monti G, Brenot F, et al. Increased interleukin-1 and interleukin-6 serum concentrations in severe primary pulmonary hypertension. Am J Respir Crit Care Med 1995; 151: 1628-1631.

3 Rabinovitch $\mathrm{M}$, Guignabert $\mathrm{C}$, Humbert $\mathrm{M}$, et al. Inflammation and immunity in the pathogenesis of pulmonary arterial hypertension. Circ Res 2014; 115: 165-175.

4 Parpaleix A, Amsellem V, Houssaini A, et al. Role of interleukin-1 receptor 1/MyD88 signalling in development and progression of pulmonary hypertension. Eur Respir J 2016; 48: 470-483.

5 Voelkel NF, Tuder RM, Bridges J, et al. Interleukin-1 receptor antagonist treatment reduces pulmonary hypertension generated in rats by monocrotaline. Am J Respir Cell Mol Biol 1994; 11: 664-675.

6 Soon E, Holmes AM, Treacy CM, et al. Elevated levels of inflammatory cytokines predict survival in idiopathic and familial pulmonary arterial hypertension. Circulation 2010; 122: 920-927.

7 Heresi GA, Aytekin M, Hammel JP, et al. Plasma interleukin-6 adds prognostic information in pulmonary arterial hypertension. Eur Respir J 2014; 43: 912-914.

8 Tian W, Jiang X, Tamosiuniene R, et al. Blocking macrophage leukotriene B4 prevents endothelial injury and reverses pulmonary hypertension. Sci Transl Med 2013; 5: 200 ra117. 
9 Kim B, Lee Y, Kim E, et al. The interleukin-1 $\alpha$ precursor is biologically active and is likely a key alarmin in the IL-1 family of cytokines. Front Immunol 2013; 4: 391.

10 Idan C, Peleg R, Elena V, et al. IL- $1 \alpha$ is a DNA damage sensor linking genotoxic stress signaling to sterile inflammation and innate immunity. Sci Rep 2015; 5: 14756.

11 Berda-Haddad Y, Robert S, Salers P, et al. Sterile inflammation of endothelial cell-derived apoptotic bodies is mediated by interleukin-1 $\alpha$. Proc Natl Acad Sci USA 2011; 108: 20684-20689.

12 Voelkel NF, Gomez-Arroyo J, Abbate A, et al. Pathobiology of pulmonary arterial hypertension and right ventricular failure. Eur Respir J 2012; 40: 1555-1565.

13 Bersudsky M, Luski L, Fishman D, et al. Non-redundant properties of IL- $1 \alpha$ and IL-1 $\beta$ during acute colon inflammation in mice. Gut 2014; 63: 598-609.

14 Dinarello CA. Interleukin-1 in the pathogenesis and treatment of inflammatory diseases. Blood 2011; 117: $3720-3732$

15 Cracowski J, Chabot F, Labarère J, et al. Proinflammatory cytokine levels are linked to death in pulmonary arterial hypertension. Eur Respir J 2014; 43: 915-917.

16 Abbate A, Kontos MC, Abouzaki NA, et al. Comparative safety of interleukin-1 blockade with anakinra in patients with ST-segment elevation acute myocardial infarction (from the VCU-ART and VCU-ART2 pilot studies). Am J Cardiol 2015; 115: 288-292.

17 Van Tassell BW, Abouzaki NA, Erdle CO, et al. Interleukin-1 blockade in acute decompensated heart failure: a randomized, double-blinded, placebo-controlled pilot study. J Cardiovasc Pharmacol 2016; 6: 544-551.

18 Van Tassell BW, Arena RA, Toldo S, et al. Enhanced interleukin-1 activity contributes to exercise intolerance in patients with systolic heart failure. PLoS One 2012; 7: e33438.

19 Ridker PM, Thuren T, Zalewski A, et al. Interleukin-1 $\beta$ inhibition and the prevention of recurrent cardiovascular events: rationale and design of the Canakinumab Anti-inflammatory Thrombosis Outcomes Study (CANTOS). Am Heart J 2011; 162: 597-605.

20 Ridker PM, Howard CP, Walter V, et al. Effects of interleukin-1 $\beta$ inhibition with canakinumab on hemoglobin Alc, lipids, C-reactive protein, interleukin-6, and fibrinogen: a phase IIb randomized, placebo-controlled trial. Circulation 2012; 126: 2739-2748.

21 Lopalco G, Rigante D, Giannini M, et al. Safety profile of anakinra in the management of rheumatologic, metabolic and autoinflammatory disorders. Clin Exp Rheumatol 2016. 